\title{
Questes
}

Revue pluridisciplinaire d'études médiévales

Journée d'étude 1 - Trier, classer, organiser | 2022 Trier, classer, organiser

\section{Aux sources de la liste. Les listes de rois du haut Moyen Âge Occidental sont-elles des documents administratifs ou des abrégés de textes d'histoire?}

\section{William Trouvé}

\section{OpenEdition}

\section{Journals}

Édition électronique

URL : https://journals.openedition.org/questes/6030

DOI : 10.4000/questes.6030

ISSN : 2109-9472

Éditeur

Les Amis de Questes

Édition imprimée

Pagination : 190-209

ISSN : 2102-7188

Référence électronique

William Trouvé, «Aux sources de la liste. Les listes de rois du haut Moyen Âge Occidental sont-elles des documents administratifs ou des abrégés de textes d'histoire? », Questes [En ligne], Journée d'étude 1 - Trier, classer, organiser | 2022, mis en ligne le 31 janvier 2022, consulté le 21 février 2022. URL : http://journals.openedition.org/questes/6030; DOI : https://doi.org/10.4000/questes.6030 


\title{
Aux sources de la liste. Les listes de rois du haut Moyen Âge Occidental sont-elles des documents administratifs ou des abrégés de textes d'histoire?
}

\author{
William TROUVÉ \\ Université d'Angers — TEMOS, CNRS UMR 9016
}

Dans son cinquième chapitre de La Raison graphique, intitulé «Que contient une liste ?», l'anthropologue Jack Goody abordait la liste comme un instrument de représentation et de contrôle du monde ${ }^{1}$. Ce procédé graphique et littéraire, consistant en une énumération de termes lexicaux ou d'items, reflète une certaine catégorisation du monde. Il offre aussi la possibilité de réordonner les items et de transformer profondément les catégories en fonction de nouvelles logiques ${ }^{2}$. Le chercheur se questionnait également sur la démarche créatrice des auteurs des listes. Son argumentaire s'appuyait sur des catalogues rassemblant des noms de souverains et de dirigeants, rédigés à diverses époques. Il rappelait que les listes sumériennes et consulaires romaines avaient servi à composer des textes annalistiques; les documents de la pratique, constitués exclusivement d'entrées courtes, étaient devenus des œuvres historiographiques plus amples. À l'inverse, de la Chronique de Gonja,

\footnotetext{
${ }^{1}$ Jack Goody, La Raison graphique. La domestication de la pensée sauvage, Paris, Les Éditions de Minuit, 1979, p. 140-196.

${ }^{2}$ Sur la liste comme représentation du monde à l'époque médiévale, voir Madeleine Jeay, Le Commerce des mots. L'usage des listes dans la littérature médiévale (XII $X V^{e}$ siècles), Genève, Droz, 2006, en particulier son premier chapitre, p. 21-55.
} 
écrite au XVIII ${ }^{\mathrm{e}}$ siècle, fut tirée une liste des rois du même nom ${ }^{3}$. Jack Goody démontrait ainsi que les listes nominales étaient soit la mise par écrit d'une expérience vécue, soit la réduction de textes plus longs. Cette réflexion mérite d'être poursuivie à partir de nouveaux exemples, qui seront extraits des sociétés altimédiévales occidentales.

Des listes de noms de rois ont en effet été produites dans les royaumes wisigothique, lombard, dans le monde anglo-saxon, et dans l'empire carolingien, du $\mathrm{VII}^{\mathrm{e}}$ au $\mathrm{IX}^{\mathrm{e}}$ siècle. Elles revêtent des formes variées : elles peuvent se limiter à l'énumération des noms, comme dans le cas lombard, ou prendre l'apparence de catalogues de règnes, à l'exemple des sources wisigothiques et franques ${ }^{4}$. L'ordre des items n'est pas arbitraire puisqu'il suit une trame chronologique. La première entrée correspond au souverain le plus ancien tandis que la dernière se rapporte au dernier roi ayant gouverné. L'ensemble produit ainsi un monde ordonné. Ces textes ont une double fonction: pratique et historiographique. Ainsi, cette succession de noms est autant un guide chronologique pour le lecteur qu'une écriture d'histoire ${ }^{5}$.

Les philologues et les historiens de l'Antiquité tardive et du haut Moyen Âge ne se sont guère attardés sur la question de la création de ces listes. Dans la foulée de Theodor Mommsen, à propos des catalogues des souverains wisigothiques, ils ont estimé que ces documents avaient été produits sinon dans les chancelleries royales, du moins avec l'accord du pouvoir royal, car elles sont presque toujours conservées avec des codes

\footnotetext{
${ }^{3}$ Ibid., p. 164-165.

${ }^{4}$ Pour une approche générale, vori David N. Dumville, « Kingship, genealogies and regnal lists » dans Early Medieval Kingship, dir. Peter H. Sawyer et Ian N. Wood, Leeds, University of Leeds, 1979, p. 72-104.

${ }^{5}$ Sur le phénomène d'évocation mémorielle sucité par les listes, voir Madeleine Jeay, "Le couple "brevitas/accumulatio" : une coexistance paradoxale », Versants, vol. 59, $\mathrm{n}^{\mathrm{o}} 1,2009$, p. 13-33.
} 
de lois ${ }^{6}$. Si l'hypothèse est recevable pour les listes lombardes, puisqu'elles constituent une partie du prologue de l'Édit de Rothari (643), elle l'est nettement moins pour les listes wisigothiques et franques. Ces dernières se présentent comme des annexes aux codes de lois dans les manuscrits où elles sont conservées; il s'agit donc de documents indépendants dont l'association avec les codes a pu être réalisée à une époque tardive par des copistes sans lien avec le pouvoir royal. Par ailleurs, des recherches récentes ont montré que des catalogues de souverains, rédigés à la même époque que les listes associées aux codes de lois, résultaient de la réduction d'un texte : ceci a été avancé pour la liste des rois vandales et alamans, qui dériverait des écrits de Victor de Tunnuna, ainsi que pour un Laterculus regum et imperatorum ad Tiberium III qui aurait été créé à partir de la Chronica Gallica ad a. $511^{7}$. Ces deux textes seraient les produits d'une mise en liste, une pratique qui consiste pour les auteurs à classer et à réécrire des informations extraites d'autres sources.

Ces remarques invitent à se demander si certaines listes de rois rédigées pendant le haut Moyen Âge sont bien des copies de documents de la pratique, élaborées à la chancellerie royale, ou si elles ont pu être

6 Theodor Mommsen, Monumenta Germaniae Historica, Scriptores, Auctores antiquissimi 13, Chronica minora, III, Berolini, Apud Weidmannos, 1898, p. 461. Il a été suivi par Luis A. García Moreno, « Sobre un nuevo ejemplar del Laterculus regum Visigothorum », Analecta Sacra Tarraconensia, 47, 1974, p. 5-14, ici p. 5 ; Francisco Bautista, «Breve historiografía: listas regias y anales en la Península Ibérica (Siglos VII-XII), Talia Dixit, 4, 2009, p. 113-190, ici p. 118-119. Sur les listes franques, voir Bruno Krusch, «Chronologica regum Francorum stirpis Merowingicae, catalogi, computationes annorum vetustae cum commentariis », dans Monumenta Germaniae Historica, Scriptores rerum Merovingicarum, VII, Hanovriae et Lipsiae, Impensis bibliopolii Hahniani, 1920, p. 476.

${ }^{7}$ Roland Steinacher, «The so-called Laterculus regum Vandalorum et Alanorum: a sixth-century african addition to Prosper Tiro's Chronicle ? », dans Vandals, Romans and Berbers. New Perspectives on Late Antique North Africa, dir. Andrew H. Merrills, Aldershot/Burlington, Ashgate, 2004, p. 163-180, ici p. 180 ; Francisco Bautista, «Dos notas sobre el ciclo historiográfico de Alfonso III », Territorio, sociedad y poder. Revista de estudios medievales, 10, 2015, p. 5-16, voir p. 12. 
composées par des copistes indépendants, à partir de sources historiographiques. Deux listes, rédigées dans des contextes différents, seront étudiées ici. Elles permettront de dégager quelques éléments de réponse sur la manière de construire ces documents, en tant que reflet d'une histoire et d'un monde.

\section{La liste des rois Wisigoths est-elle la copie d'un document de la pratique ou un abrégé de l'Histoire des Goths d'Isidore de} Séville?

Depuis la défaite de Vouillé en 507, le royaume des Wisigoths est resserré dans la péninsule Ibérique, malgré un léger débordement en Septimanie, région autour de Narbonne. L'unification territoriale, par l'annexion du territoire suève sous le règne de Léovigild (569-586) et des dernières enclaves byzantines vers 624, ainsi que l'unité religieuse, instaurée au moment de la conversion au catholicisme de Récarède en 589, ont permis aux souverains de consolider leur position dans cet espace. En raison de la nature élective du pouvoir, les successions au trône ont néanmoins été marquées par de nombreux coups de force et ont pu donner l'impression d'une instabilité politique qui aurait logiquement conduit à la chute du royaume par les troupes arabo-musulmanes, venues du Maghreb pour conquérir 1'Hispania, en 711. Cette interprétation est aujourd'hui dépassée, et les chercheurs sont enclins à réévaluer les sources produites dans les derniers temps du monde wisigothique ${ }^{8}$. Monarchie théocratique, puisque les évêques ont autant leur place autour du souverain que les laïcs, et dernière expression de la romanité, tant dans

\footnotetext{
${ }^{8}$ Notamment, Pablo Povedas Arias, «Relectura de la supuesta crisis del fin del reino visigodo de Toledo : una aproximación al reinado de Egica a través de sus fuentes legales », Anuario de historia del derecho español, vol. 1, $\mathrm{n}^{\circ} 85,2015$, p. 14-46.
} 
ses structures administratives que dans les écrits de ses érudits ${ }^{9}$, le royaume des Wisigoths fait figure d'originalité dans l'Europe occidentale du VII ${ }^{\mathrm{e}}$ siècle.

C'est dans ce contexte que doit être interprétée la création de la liste des rois wisigoths. Celle-ci, plus connue sous sa dénomination latine de Laterculus regum Visigothorum, se rencontre généralement avec des exemplaires du Liber Iudiciorum, code de lois publié sous Réceswinthe (649-672) en 654, dont l'une des finalités était de légitimer le souverain exercice $^{10}$. Les plus anciennes copies de la liste sont également celles du code législatif, ce qui laisse penser que ces deux œuvres ont été rédigées ensemble. Les études portant sur la liste des rois des Wisigoths n'ont jamais traité de la question des sources employées dans sa rédaction. Nous nous proposons ici d'étudier cette question. En prolongeant le postulat précédent, peut-on envisager cette liste de règnes comme l'abrégé de textes d'histoire ou bien la copie d'un registre administratif ? La fixation des noms aurait été effectuée soit à partir da la réduction de textes historiographiques, soit par les fonctionnaires royaux au fur et à mesure des successions au trône. Afin de répondre à cette question, il faut questionner les différentes copies conservées du catalogue. Or celles-ci ne coïncident pas toujours, comme le révèle une rapide description des trois témoins les plus anciens.

\footnotetext{
${ }^{9}$ Pour une approche générale de l'histoire wisigothique, voir Herwig Wolfram, Histoire des Goths, Paris, Albin Michel, 1990 ; Adeline Rucquoi, Histoire médiévale de la péninsule Ibérique, Paris, Seuil, 1993, p. 36-73; Roger Collins, Visigothic Spain, Oxford, Blackwell Publishing, 2004.

10 Thomas Deswarte, «Le code du roi Réceswinthe (654) a-t-il abrogé les droits antérieurs ? », dans Traditio Juris. permanence et/ou discontinuité du droit romain durant le haut Moyen Age, dir. Alain Dubreucq et Christian Lauranson-Rosaz, Lyon, Cahiers du Centre d'histoire médiévale, 3, 2005, p. 57-76; Céline Martin, « La réforme visigothique de la justice : les années Recceswinth », dans Derecho y justicia. El poder en la Europa medieval, dir. Nilda Guglielmi et Adeline Rucquoi, Buenos Aires, CONICET CNRS, 2008, p. 37-57 ; Michael J. Kelly, « Le Liber Iudiciorum de Recceswinth: histoire, récit et sens », Visigothic Symposium, 1, 2016-2017, p. 110134.
} 
Le manuscrit Vatican, BAV, Reg. lat. 1024, copié dans la région d'Urgell ou de La Cerdagne, contient sur le verso de son dernier folio (fol. 138) une liste de rois fragmentaire, rédigée au début du VIII ${ }^{\mathrm{e}}$ siècle $^{11}$. Les premières lignes sont effacées. Seuls trente items sont encore lisibles. Le texte débute avec Athaulf (410-416) (cap. 8), deuxième souverain wisigothique, et s'achève prématurément avec Tulga (639-642) (c. 37) ${ }^{12}$. Il s'agit de la seule copie à évoquer la régence ostrogothique de Théodoric le Grand (511-526) sur deux items (c. 17-18) ainsi que l'association au trône de Léovigilde (569-586), lors de la deuxième année du règne de son frère Liuva $\mathrm{I}^{\mathrm{er}}(568-573)$ (c. 27).

Le manuscrit Paris, BnF, lat. 4668 a été compilé dans le troisième tiers $\mathrm{du} \mathrm{IX}^{\mathrm{e}}$ siècle, probablement en Narbonnaise selon Bernhard Bischoff ${ }^{13}$. La liste, copiée directement après la table des matières du Liber Iudiciorum, se compose d'une quarantaine d'items. Il s'agit de la plus longue conservée. Elle s'ouvre par une introduction sur l'errance puis l'arrivée des Goths en Hispanie (c. 1-4) et se conclut par une série de petites notices concernant les morts de Chindaswinte et de Réceswinthe ainsi que les onctions de Wamba (672) et d'Ervige (680) (c. 40-47). Elle est également le seul témoin à préciser que Gésalic (507-511) (c. 16) régna une année en dehors de l'Hispanie et qu'il y eut une vacance du pouvoir de cinq mois après le règne d'Athanagilde (551-568) (c. 24).

\footnotetext{
${ }^{11}$ La meilleure étude de ce manuscrit est celle de Yolanda García López, Estudios críticos y literarios de la Lex wisigothorum, Alcalá de Henares, Universidad de Alcalá, 1996, p. 41-43.

${ }^{12}$ Le texte a été édité par Theodor Mommsen, Chronica minora, op. cit., p. 464-469.

13 Bernhard Bischoff, Katalog der festländischen Handschriften des neunten Jahrhunderts (mit Ausnahme der wisigotischen), III : Padua-Zwickau, Wiesbaden, Harrassowitz, 2014, p. $100 \mathrm{n}^{\mathrm{0}} 4325$.
} 
Enfin, le manuscrit Paris, $\mathrm{BnF}$, lat. 4667 a été rédigé dans la région de Gérone, vers $827^{14}$. Le catalogue des souverains, qui précède le code législatif, a été copié sur le recto du septième feuillet. Une introduction, presque identique au témoin précédent, débute la copie. Les entrées qui suivent sont toutes construites sur le modèle $A$ regnauit $X$ annos $Y$ menses. Les mentions d'Achila et d'Ardo, souverains wisigothiques reconnus seulement en Tarraconaise dans les années 710, précèdent l'expression et fuerunt reges Gotorum qui regnauerunt $X L$, qui ferme le texte.

Ces variantes témoignent de réécritures propres à chaque copie. Les scribes successifs ont vraisemblablement employé plusieurs sources au moment de la transcription de ces textes. Ceci invite à se questionner sur les documents qui ont pu être employés au moment de la rédaction de l'archétype de la liste, en étudiant les sources historiographiques du VII siècle.

La liste des rois rappelle la version longue de l'Historia Gothorum, rédigée par Isidore de Séville en 624. Ce texte se compose de notices biographiques relatives à chaque souverain wisigothique, depuis le premier d'entre eux, Athanaric ( $\mathrm{v}^{\mathrm{e}}$ siècle), jusqu'au début du règne de Sisenand (631-636) ${ }^{15}$. Plusieurs durées de règne y sont indiquées. L'œuvre du Sévillan et la liste ont-elles des liens ? Les premiers items de la liste pourraient-ils être les abrégés du texte d'Isidore plutôt que la copie d'un registre administratif? Une étude comparée des trois listes avec l'Historia s'impose, depuis le règne d'Athanaric jusqu'à celui de Récarède II, le prédécesseur de Sisenand, ce qui correspond à la trame

\footnotetext{
${ }^{14}$ Ibid., p. 55-60 ; Jesús Alturo Perucho, « El Liber iudicum manuscrito latino 4667 de la biblioteca nacional de Francia. Análisis paleográfrico », Historia instituciones, documentos, 30, 2003, p. 9-54.

${ }^{15}$ Voir l'étude et l'édition critique de Cristóbal Rodríguez Alonso, Las historias de los Godos, Vandalos y Suevos de Isidoro de Sevilla. Estudio, edición critica y traducción, León, Centro de estudio e investigacion « San Isidoro », 1975.
} 
chronologique isidorienne et à vingt-huit entrées (c. 6-33) du catalogue de rois.

Neuf passages de la liste correspondent au texte isidorien (c. 6, 7, $8,10,11,15,17,19,28)$. Onze entrées de la liste sont proches de l'Histoire des Goths, soit une bonne moitié des éléments comparés. Trois d'entre elles rendent compte, de façon identique, des conditions de règne d'un souverain (c. 16, 18, 27), mais les formulations diffèrent entre les sources. Les huit autres (c. 20, 21, 22, 24, 29, 30, 31, 32) concernent les durées de règne, dont le degré de précision est plus haut dans le catalogue des rois, puisque les mois et parfois les jours sont indiqués, tandis que l'Histoire des Goths cite uniquement les années. Sur ces vingthuit entrées, les trois quarts rappellent donc le récit d'Isidore de Séville. Néanmoins, huit passages sont différents (c. 9, 12, 13, 14, 23, 25, 26, 33), ce qui empêche d'envisager la liste comme un abrégé d'histoire isidorienne. Les deux œuvres partagent indéniablement des liens textuels, mais il est probable que les auteurs des listes aient puisé des informations dans plusieurs sources, parmi lesquelles l'Historia Gothorum ou un texte commun au catalogue et au récit du Sévillan.

Les autres textes historiographiques wisigothiques - la chronique de Jean de Biclar en particulier - sont éloignés de la liste des rois des Wisigoths. De fait, il semble possible d'envisager ce document comme la copie dérivée d'un registre administratif, dans lequel les fonctionnaires royaux auraient consigné les noms des souverains, à l'image de ce qui était pratiqué dans le monde romain ${ }^{16}$. Néanmoins, cette origine reste une hypothèse parmi d'autres, compte tenu du silence de la documentation.

\footnotetext{
${ }^{16}$ Brian Croke, « City chronicles of Late Antiquity », dans Reading the Past: the Past in Late Antiquity, dir. Brian Croke et al., Ruschcutters Bay, Australian National University Press, 1990, p. 165-203 ; Richard W. Burgess, The Chronicle of Hydatius and the Consularia Constantinopolitana. Two Contemporary Accounts of the Final Years of the Roman Empire, Oxford, Clarendon Press, 1993, p. 175-212.
} 
En revanche, il est certain que la diffusion de cette liste s'est accompagnée de modifications scripturales successives. Les copistes ont parfois cherché à en préciser la chronologie ou ont ajouté des événements marquants de l'histoire tolédane. Puisqu'ils avaient à disposition l'Historia Gothorum, ils ont indiqué la date d'accession au pouvoir de Léovigilde, comme dans le manuscrit du Vatican, ou mentionné la retraite de Gésalic, comme dans le manuscrit Paris, lat. 4668. Le texte isidorien, parce qu'il fait autorité, a été exploité pour rectifier le document mineur qu'était la liste afin de réordonner le monde wisigothique.

\section{La pratique de la mise en liste : le cas du Paris, BnF, lat. 2718}

Le processus de mise en liste est plus évident dans le cas du manuscrit Paris, BnF, lat. 2718. Le codex, rédigé initialement dans les années 830 à Saint-Martin de Tours, fut élaboré en plusieurs temps, comme en témoigne la disposition discontinue de certains textes, qui remplissent d'anciens blancs, ainsi que la présence de différentes encres. Il s'agit probablement d'un manuscrit destiné à un usage personne ${ }^{17}$. Il contient principalement des sermons de saint Augustin ainsi que des capitulaires et des modèles d'actes carolingiens, connus sous le nom de Formulae imperiales. La liste, qui se trouve sur le recto du premier feuillet, fut copiée au plus tard au $\mathrm{x}^{\mathrm{e}}$ siècle, comme l'écriture caroline du texte, marquée par une semi-onciale propre à l'abbaye tourangelle, le laisse envisager ${ }^{18}$.

17 Hubert Mordek, Bibliotheca capitularium regum Francorum manuscripta; Überlieferung und Traditionszusammenhang der fränkischen Herrschererlasse, München, Monumenta Germaniae Historica, 1995, p. 422-430 ; Alice Rio, Legal Practice and the Written Word in the Early Middle Ages: Frankish Formulae, c. 5001000, Cambridge, Cambridge University Press, 2009, p. 252-254.

18 Bernhard Bischoff, Paléographie de l'Antiquité romaine et du Moyen Âge occidental, Paris, Picard, 1993, p. 131. 
Il ne s'agit pas d'une liste à proprement parler. Les items, jamais isolés par des renvois à la ligne, ont une disposition continue. Toutefois, la composition et le contenu de l'œuvre amènent à la classer parmi les listes. Elle est constituée d'une suite de propositions courtes, construites le plus souvent sur le même modèle : nom du roi, indication de sa parenté avec le souverain précédent, durée de règne. Les propositions débutent soit par mortuo, soit par post, ce qui crée un rythme anaphorique dans un style déjà énumératif. Il y a donc un «effet liste ${ }^{19}$ ». Quant au contenu, il consiste en une description de la dynastie mérovingienne, par branche et sous-branche, ainsi qu'en un petit traité chronologique, dans lequel sont indiquées les durées de chaque règne. Le document emprunte donc autant à la généalogie qu'aux catalogues de souverains, qui sont des genres énumératifs ${ }^{20}$.

Une comparaison de cette liste avec d'autres sources mérovingiennes et carolingiennes dévoile la démarche de l'auteur, un moine de Saint-Martin probablement, pour construire son texte. Il a d'abord utilisé l'œuvre de Grégoire de Tours ${ }^{21}$. Les deux premières entrées sont des passages légèrement retouchés du deuxième livre de l'Histoire des Francs (tableau 1).

\footnotetext{
${ }^{19}$ Voir à ce sujet Liste et effet liste en littérature, dir. Sophie Milcent-Lawson, Michelle Lecolle et Raymond Michel, Paris, Classiques Garnier, 2013, en particulier les réflexions de Georges Molinié, « Vers une sémiotique de la liste », p. 565-570.

${ }^{20}$ Voir les définitions de Léopold Génicot, Les Généalogies, Turnhout, Brepols, 1975, p. 11-12 et de David N. Dumville, « Kingship », art. cit., p. 97.

${ }^{21}$ Composées à Tours à la fin du $\mathrm{VI}^{\mathrm{e}}$ siècle, les Histoires y sont encore recopiées dans le courant du $\mathrm{IX}^{\mathrm{e}}$ siècle, comme l'indique une étude de la tradition manuscrite de l'œuvre. Voir Pascale Bourgain et Martin Heinzelmann, «L'œuvre de Grégoire de Tours : la diffusion des manuscrits », dans Grégoire de Tours et l'espace gaulois. Actes du congrès international (Tours, 3-5 novembre 1994), dir. Nancy Gauthier et Henri Galinié, Tours, Fédération pour l'édition de la Revue archéologique du Centre de la France, 1997, p. 274, 279, 288 et 292.
} 


\section{Tableau 1}

Comparaison entre la liste du manuscrit Paris, lat. 2718

et l'Histoire des Francs de Grégoire de Tours

\begin{tabular}{|c|c|}
\hline & Histoire de \\
\hline $\begin{array}{l}\text { 1. In consularibus namque legitur } \\
\text { Teodemirum regem francorum filium } \\
\text { RIGIMIRI; }\end{array}$ & $\begin{array}{l}\text { Nam et in consolaribus legimus, } \\
\text { Theudomerem regem Francorum, filium } \\
\text { Richimeris quondam }{ }^{23} \text { (Grég., Hist., II, 9) }\end{array}$ \\
\hline $\begin{array}{l}\text { 2. De huius stirpe quidam Meroueum } \\
\text { regem fuisse adserunt cuius fuit filius } \\
\text { Hildericus rex Francorum. }\end{array}$ & $\begin{array}{l}\text { De huius stirpe quidam Merovechum regem } \\
\text { fuisse adserunt, cuius fuit filius Childericus. } \\
\text { (Grég,. Hist., II, 9) }\end{array}$ \\
\hline
\end{tabular}

Poursuivant la lecture du texte de Grégoire de Tours jusqu'au règne de Childebert II (†595) (c. 17), l'auteur de la liste en a extrait des informations dynastiques et chronologiques (tableau 2). Il a classé et réécrit chaque règne de façon à obtenir une série de propositions très courtes.

\section{Tableau 2}

Comparaison entre la liste du manuscrit Paris, lat. 2718, l'Histoire des Francs de Grégoire de Tours et la Chronique de Frédégaire (suite)

\begin{tabular}{|l|l|}
\hline $\begin{array}{l}\text { 3. Mortuo Hilderico, Hlodoueus filius } \\
\text { eius regnauit annos XXX. }\end{array}$ & $\begin{array}{l}\text { His gestis, mortuo Childerico, } \\
\text { regnauit Chlodovechus, filius eius (Grég., } \\
\text { Hist., II, 27) }\end{array}$ \\
\hline
\end{tabular}

22 Il s'agit de notre propre transcription du texte. Une édition avait été établie par André Duchesne, Historiae Francorum scriptores coaetanei, vol. 1, Paris, s. n., 1636, p. 793, rééd. dans Martin Bouquet, Recueil des historiens des Gaules et de la France, vol. 2, Paris, V. Palmé, 1869, p. 695.

${ }^{23}$ Édité dans Bruno Krusch et Wilhelm Levison, Monumenta Germaniae Historia, Scriptores rerum Merovingicarum, I, pars. 1, Gregorii Episcopi Turonensis Libri Historiarum X, Hanovriae, Impensis Bibliopolii Hahniani 1937-1951, p. 1-537. 


\begin{tabular}{|c|c|}
\hline & $\begin{array}{l}\text { Fueruntque omnes dies regni eius anni } \\
\text { triginta (Grég., Hist., II, 43) }\end{array}$ \\
\hline $\begin{array}{l}\text { 4. Post eum quatuor filii eius inter se } \\
\text { diuiserunt regnum } \quad \text { Teodericus, } \\
\text { Hlodomirus, Hildebertus adque } \\
\text { Hlotarius. }\end{array}$ & $\begin{array}{l}\text { Defuncto igitur Chlodovecho regi, } \\
\text { quattuor filii eius, id est Theudoricus, } \\
\text { Chlodomeris, Childeberthus atque } \\
\text { Chlothacharius, regnum eius accipiunt et } \\
\text { inter aequa lantia dividunt (Grég., Hist., } \\
\text { III, 1) } \\
\text { Quattuor filii Clodoveo, id est } \\
\text { Theudericus, Chlodomeres, Childebertus } \\
\text { et Chlotarius, regnum eius aequo ordine } \\
\text { inter se deviderunt (Fred., Chron., III, 29) }\end{array}$ \\
\hline 5. Teodericus regnauit annos XXIII. & $\begin{array}{l}\text { Theudoricus non post multos dies obiit } \\
\text { vicinsimo tertio regni sui anno (Grég., } \\
\text { Hist., III, 23). }\end{array}$ \\
\hline $\begin{array}{l}\text { 6. Post eum regnauit Teutbertus filius } \\
\text { eius annos XIIII. }\end{array}$ & $\begin{array}{l}\text { Habebat iam tunc Theudoricus filium } \\
\text { nomen Theudobertum (Grég., Hist., III, 1) } \\
\text { Mortuo ergo Theudoberto quarto decimo } \\
\text { regni sui, regnauit theudoaldus, filius } \\
\text { eius (Grég., Hist., III, 37) }\end{array}$ \\
\hline $\begin{array}{l}\text { 7. Mortuo Theutberto, regnauit } \\
\text { Theodoaldus filius eius annos VII. }\end{array}$ & $\begin{array}{l}\text { de [Deuteria] parvolum filium habebat } \\
\text { Theodobaldum nomen [Theudobertus] } \\
\text { (Grég., Hist., III, 27) } \\
\text { septimo regni sui anno mortuos est } \\
\text { (Grég., Hist., IV, 9) }\end{array}$ \\
\hline $\begin{array}{l}\text { 8. Mortuo Theodoaldo, Hlotharius frater } \\
\text { Teoderici regnum eius accepit. }\end{array}$ & $\begin{array}{l}\text { Regnumque eius Chlothacharius rex } \\
\text { accepit (Grég., Hist., IV, 9) }\end{array}$ \\
\hline $\begin{array}{l}\text { 9. Defuncto autem Hlodomiro, eius } \\
\text { regnum Hildebertus et Hlotharius inter } \\
\text { se diuiserunt. }\end{array}$ & I \\
\hline 10. Et mortuo Hildeberto regnum eius & Childeberthus igitur re \\
\hline
\end{tabular}




\begin{tabular}{|c|c|}
\hline $\begin{array}{l}\text { Hlotharius germanus suus accepit. } \\
\text { 11. Qui predictus Hildebertus regnauit } \\
\text { annos XLVI }\end{array}$ & (...) obiit. (Grég., Hist., IV, 20) \\
\hline $\begin{array}{l}\text { 12. Hlotharius uero germanus Hildeberti } \\
\text { regnauit annos LI. }\end{array}$ & $\begin{array}{l}\text { Rex vero Chlotharius anno } \\
\text { quinquaginsimo primo regni sui cum } \\
\text { multis muneribus limina beati Martini } \\
\text { expetiit (Grég., Hist., IV, 21) } \\
\text { Chlothariius... } 51 \text { regni sui anno vexatus } \\
\text { a febre obiit. (Fred., Chron., III, 55) }\end{array}$ \\
\hline $\begin{array}{l}\text { 13. Cui successerunt filii quatuor in } \\
\text { regno quorum nomina hec sunt } \\
\text { Haribertus, Guntramnus, Hilpericus et } \\
\text { Sigibertus. }\end{array}$ & $\begin{array}{l}\text { Et sic inter se hii quattuor, id est } \\
\text { Chariberthus, Gunthramnus, Chilpericus } \\
\text { atque Sigiberthus, divisionem legitimam } \\
\text { faciunt (Grég., Hist., IV, 22) }\end{array}$ \\
\hline $\begin{array}{l}\text { 14. Sed numerus annorum Hariberti et } \\
\text { Hilperici nec gesta declaratur. }\end{array}$ & / \\
\hline $\begin{array}{l}\text { 15. Guntramnus uero regnauit annos } \\
\text { XXXIII. }\end{array}$ & $\begin{array}{l}\text { Anno } 33 \text { regni Gunthramni. Eo anno } \\
\text { quinto Kal. Aprilis ipse rex moritur } \\
\text { (Fred., Chron., IV, 14) }\end{array}$ \\
\hline $\begin{array}{l}\text { 16. Sigibertus autem regnauit annos } \\
\text { XIIII. }\end{array}$ & $\begin{array}{l}\text { Obiit [Sigyberthus] autem quarto decimo } \\
\text { regni sui anno aetate quadraginaria } \\
\text { (Grég., Hist., IV, 51) }\end{array}$ \\
\hline $\begin{array}{l}\text { 17. Mortuo autem Sigiberto, Hildebertus } \\
\text { filius eius regnauit pro eo annos XXII. }\end{array}$ & $\begin{array}{l}\text { Mortuo autem Sigybertho, regnauit } \\
\text { Childeberthus, filius eius, pro eo (Grég., } \\
\text { Hist., IV, 51) }\end{array}$ \\
\hline
\end{tabular}

Dans ce premier ensemble d'items, la neuvième entrée, concernant Clodomir, pourrait être liée au livre III, c. 6, qui mentionne également la mort du souverain. Mais le partage territorial évoqué ensuite dans la liste n'apparaît pas chez l'évêque tourangeau. Il peut donc s'agir d'une interprétation de la part du scribe de Saint-Martin. 
Puis, dès le c. 15 et systématiquement à partir du c. 18, l'auteur utilise la Chronique de Frédégaire (tableau 3), car l'Histoire des Francs s'achève vers 591-594. Cette césure explique pourquoi la durée de règne de Childebert II (c. 17), mort en 595, n'est pas indiquée. L'information n'apparaît pas chez Frédégaire, qui, de toute façon, a pour source l'Histoire de Grégoire de Tours. L'auteur de la liste n'a pas non plus utilisé le Liber historiae Francorum, composé vers 727 en Neustrie, qui, dans sa recension B, octroie pourtant vingt années à Childebert (c. 37). Cette absence signifie que le scribe, soit n'avait pas connaissance du Liber, soit l'a rejeté de ses sources.

La version de la Chronique utilisée pour rédiger la liste devait se terminer avec Dagobert III (711-715), comme le suggère la rupture de style à partir du c. 30. Le moine a employé cette source en suivant une méthode identique à celle utilisée pour l'exploitation de l'Historia. Il a classé, organisé et réécrit les informations qui répondaient à ses préoccupations intellectuelles.

\section{Tableau 3}

Comparaison entre la liste du manuscrit Paris, lat. 2718

et la Chronique de Frédégaire

\begin{tabular}{|c|c|}
\hline Paris, BnF, lat. 2718 & Chronique de Frédégaire ${ }^{24}$ \\
\hline $\begin{array}{l}\text { 18. Mortuo namque Hildeberto, } \\
\text { Teutbertus et Teodericus filii eius regnum } \\
\text { ipsius adsumunt. }\end{array}$ & $\begin{array}{l}\text { Quarto anno, post quod Childebertus } \\
\text { regnum Guntramni acciperat, defunctus } \\
\text { est ; regnumque eius filii sui Teudebertus } \\
\text { et Teudericus adsumunt. (Fred., Chron., } \\
\text { IV, 16) }\end{array}$ \\
\hline
\end{tabular}

${ }^{24}$ Édité dans Bruno Krusch, Monumenta Germaniae Historica, Scriptores rerum Merovingicarum, tomus II, Fredegarii et aliorum Chronica. Vitae Sanctorum, Hanovriae, Impensis Bibliopolii Hahniani, 1888, p. 18-193. 


\begin{tabular}{|c|c|}
\hline 19. Teutbertus regnauit annos XVII. & I \\
\hline 20. Teodericus regnauit annos XVIII. & $\begin{array}{l}\text { [Theudericus] contra Chlotharius } \\
\text { exercitum anno XVIIII regni sui de Auster } \\
\text { et Burgundias mouere precepit (Fred., } \\
\text { Chron., IV, 38) } \\
\text { Ipso quoque anno (...) Theudericus Mettis } \\
\text { profluuium uentris moritur (Fred., } \\
\text { Chron., IV, 39). }\end{array}$ \\
\hline $\begin{array}{l}\text { 21. Hlotarius filius Hilperici interfectis } \\
\text { duobus filiis Teoderici, Sigiberto et } \\
\text { Hildeberto, regnum quod tenuit } \\
\text { Teodericus, pleniter in suam redegit } \\
\text { potestatem et regnauit XLV. }\end{array}$ & $\begin{array}{l}\text { Sigybertus et Corbus, filius Theuderici, } \\
\text { iusso Clothariae interfecti sunt. (Fred., } \\
\text { Chron., IV, 42) } \\
\text { Anno } 46 \text { regni sui Chlotharius moritur } \\
\text { (Fred., Chron., IV, 56) }\end{array}$ \\
\hline $\begin{array}{l}\text { 23. Cui successu [sic] in regno eius filius } \\
\text { nomine Dagobertus qui regnauit annos } \\
\text { XVI. }\end{array}$ & $\begin{array}{l}\text { Anno sexto decemo regni sui Dagobertus } \\
\text { (...) aegrotare cepit. (...) post paucus dies } \\
\text { Dagobertus amisit spiritum. (Fred., } \\
\text { Chron., IV, 79) }\end{array}$ \\
\hline $\begin{array}{l}\text { 24. Mortuo Dagoberto, Hlodoueus filius } \\
\text { eius regnauit annos XVIIII. }\end{array}$ & $\begin{array}{l}\text { Chlodoueues, filius Dagoberti (...), } \\
\text { regnauitque annis } 18 \text { (Fred., Cont., 1) }\end{array}$ \\
\hline $\begin{array}{l}\text { 25. Post ipsum uero successit filius eius } \\
\text { in regno nomine Sigibertus qui regnauit } \\
\text { annos } X \text {. }\end{array}$ & I \\
\hline $\begin{array}{l}\text { 26. Iterum tertius Hlotarius filius } \\
\text { Hlodouei regnauit annos IIII. }\end{array}$ & $\begin{array}{l}\text { His diebus Chlotharius rex a valida febre } \\
\text { correptus, obiit in iuventute regnauitque } \\
\text { annis } 4 \text { (Fred., Cont., 2) }\end{array}$ \\
\hline $\begin{array}{l}\text { 27. Cui successit in regno Teodericus } \\
\text { frater eius qui regnauit annos XVII. }\end{array}$ & $\begin{array}{l}\text { Theudericus vero, frater eius, in regno } \\
\text { successit (Fred, Cont., 2) } \\
\text { Mortuus est autem Theudericus rex; } \\
\text { regnauit autem ann. 17. (Fred., Cont., 6) }\end{array}$ \\
\hline $\begin{array}{l}\text { 28. Quo mortuo Hlodeueus filius eius } \\
\text { successit in regno et regnauit annos IIII. }\end{array}$ & $\begin{array}{l}\text { Chlodovecho, filio eius parvulo, elegerunt } \\
\text { in regnum. Non post multos enim annos } \\
\text { praedictus rex Chlodoveus egrotans }\end{array}$ \\
\hline
\end{tabular}




\begin{tabular}{|l|l|}
\hline & $\begin{array}{l}\text { mortuus est; regnauit autem ann. } 4 . \\
\text { (Fred., Cont., 6) }\end{array}$ \\
\hline $\begin{array}{l}\text { 29. Post ipsum uero Hildebertus frater } \\
\text { eius regnauit annos XVI. }\end{array}$ & $\begin{array}{l}\text { Childebertus, frater eius, in regnum } \\
\text { resedit. (Fred, Cont., 6) } \\
\text { Mortuus est autem his diebus } \\
\text { Childebertus rex (...); regnauit autem } \\
\text { ann. 16. (Fred., Cont., 7) }\end{array}$ \\
\hline $\begin{array}{l}\text { 30. Et post ipsum Dagobertus filius eius } \\
\text { accepit regnum patris sui et regnauit } \\
\text { annos V. }\end{array}$ & $\begin{array}{l}\text { Dagobertus, filius eius, sedem regni } \\
\text { patris sui accepit. (Fred., Cont., 7) } \\
\text { Itidem tempore Dagobertus rex obiit ; } \\
\text { regnauit itaque an. 5. (Fred., Cont., 9) }\end{array}$ \\
\hline
\end{tabular}

Les sources de deux items ne sont pas identifiées. L'auteur de la liste attribue une durée de règne à Théodebert (c. 19), ce que ne font ni l'évêque tourangeau (IV, 50) ni Frédégaire (IV, 38). Il est possible que le moine de Saint-Martin ait lu de façon très précise les textes et ait obtenu la durée du règne de Théodobert par un calcul chronologique. De la même façon, Grégoire de Tours et Frédégaire ne font pas mention du règne de Sigebert III (c. 25). Seules les listes de rois qui accompagnent la Loi salique le mentionnent mais en lui attribuant une durée de règne de 23 années au lieu de $10^{25}$. L'emploi de ce type de document n'est pas impossible, comme le prouve la suite du document.

Du c. 31 jusqu'à la fin du texte, l'auteur a en effet remployé un catalogue de souverains sans le modifier (tableau 4). Ce catalogue se retrouve dans deux exemplaires de la Loi salique, qui appartiennent à la classe E, une révision effectuée sous Charlemagne vers $798^{26}$.

\footnotetext{
${ }^{25}$ Bruno Krusch, «Chronologica regum Francorum... », op. cit. (voir n. 6), p. 480481.

${ }^{26}$ Paris, BnF, lat. 4409 ; Vatican, BAV, Reg. lat. 846. Karl A. Eckhardt, Monumenta Germaniae Historica, Legum sectio I, Legum Nationum Germanicarum, IV, pars I, Pactus Legis Salicae, Hanovriae, Impensis Bibliopolii Hahniani, 1962, p. 40. Récemment, une autre datation, en 789, a été proposée par Karl Ubl, «Die erste
} 


\section{Tableau 4}

Comparaison entre la liste du manuscrit Paris, BnF, lat. 2718

et la liste des rois Francs (Loi salique, classe E)

\begin{tabular}{|c|c|}
\hline Paris, BnF, lat. 2718 & Liste de rois francs $^{27}$ \\
\hline 31. Dagobertus annos IIII obiit in $V$. & $\begin{array}{l}\text { Dagobertus regnauit annos IIII obiit in } \\
\text { quinto. }\end{array}$ \\
\hline 32. Hilpericus regnauit annos $V$. & Hilpericus regnauit annos $V$. \\
\hline 33. Teodericus regnauit annos XVII. & Theodericus regnauit annos XVII. \\
\hline $\begin{array}{l}\text { 34. Karolus sine alio rege imperauit } \\
\text { annos VII. }\end{array}$ & $\begin{array}{l}\text { Charolus sine alio rege imperauit annos } \\
\text { VII. }\end{array}$ \\
\hline 35. Hildericus regnauit annos [...]. & Childricus regnauit annos VIIII. \\
\hline $\begin{array}{l}\text { 36. Pippinus regnauit annos XVI et } \\
\text { dimidium. }\end{array}$ & Pippinus regnauit annos XVI et dimedio. \\
\hline
\end{tabular}

Ces analyses comparatives permettent de dégager l'une des méthodes du faiseur de liste. Comme le suggère le c. 14, le moine de Saint-Martin a effectué un travail de lecture des sources, de recherche et d'identification des informations qui l'intéressaient. Il a ensuite extrait, réduit et reformulé ces renseignements. Enfin, il a ordonné le produit de cette réécriture avant de lui adjoindre un matériau brut, qui termine son texte. Il propose donc un abrégé d'histoire mérovingienne, à partir de textes plus amples. Surtout, il envisage un ordre du monde mérovingien selon une perception généalogique.

La présence d'une liste de rois dans un manuscrit qui relève du droit et du spirituel n'a rien de surprenant puisque d'autres en font état ${ }^{28}$.

Leges-Reform Karls des Großen », dans Das Gesetz - The Law - La loi, dir. Guy Duldentops et Andres Speer, Berlin/New-York, De Gruyter, 2014, p. 82-88. La datation traditionnelle, proposée par K. A. Eckhardt, reste majoritairement acceptée.

${ }^{27}$ Bruno Krusch, « Chronologica regum Francorum... », op. cit. (voir n. 6), p. 481. 
En revanche, que le catalogue ait trait aux souverains mérovingiens alors que les documents juridiques émanent de la chancellerie carolingienne étonne davantage. Ils n'ont a priori aucun lien. Pour déterminer en quoi l'ajout de ce court texte était nécessaire pour le moine de Saint-Martin, il faut comprendre la lecture qu'il faisait de son manuscrit au tournant du $\mathrm{x}^{\mathrm{e}}$ siècle, époque de rédaction de la liste. En raison de leur datation haute, les formules d'actes et les capitulaires conservés étaient probablement moins envisagés en fonction de leur finalité pratique que de leurs apports historiques $^{29}$. Le manuscrit contient l'Ordinatio imperii, un capitulaire de 817, qui règle la succession de Louis le Pieux entre ses fils d'alors. Le capitulaire peut aussi être lu comme un traité sur le partage du pouvoir ${ }^{30}$. La thématique de ce texte renvoie alors à celle du catalogue, puisque tous deux traitent des successions royales. Le moine de Saint-Martin aurait composé une liste de souverains mérovingiens afin de retracer la généalogie des rois francs depuis les origines. Pourquoi ne pas avoir actualisé le document jusqu'à l'époque de rédaction du catalogue plutôt que l'arrêter à Pépin? Cette absence laisse penser que le moine connaissait suffisamment l'histoire carolingienne, mais qu'il était bien plus en peine pour maîtriser les différents règnes mérovingiens. La création de ce texte serait alors une réponse à un besoin d'érudition dans un codex apprécié comme une source de réflexions historiques et spirituelles.

${ }^{28}$ Il serait trop long de tous les énumérer. À titre d'exemple pour le monde carolingien, peuvent être cités le Wolfenbüttel, HAB, Cod. Guelf. 97 Weiss, qui contient une loi salique et une liste de rois mérovingiens, ou encore le Vatican, BAV, Reg. lat. 1024, qui conserve un catalogue de souverains francs et un exemplaire du Bréviaire d'Alaric.

${ }^{29}$ Sur la copie des formulae mérovingiennes à l'époque carolingienne, voir les remarques d'Alice Rio, Legal practice ..., op. cit., p. 169.

${ }^{30}$ L'édition de référence est celle d'Alfred Boretius, Victor Kraus, Monumenta Germaniae Historica, Legum sectio II. Capitularia regum Francorum, t. 1, Hanovriae, Impensis Bibliopolii Hahniani, 1883 p. 270-273. 
Deux démarches président à l'ordonnancement du monde dans les listes de noms de rois du haut Moyen Âge: la mise par écrit d'une expérience vécue ou la réduction de textes amples. Réussir à les identifier est une entreprise difficile tant les listes conservées ont été retouchées. Le cas wisigothique a montré que les catalogues de souverains pourraient initialement procéder d'une pratique administrative. Ils refléteraient alors l'expérience vécue des fonctionnaires en charge de mettre à jour les registres royaux. La liste nominale appartiendrait aux documents de la pratique. Il faut toutefois reconnaître que, parmi les listes de cette époque qui nous ont été transmises, la très grande majorité relève d'une fonction historiographique. Ceci explique pourquoi les scribes se sont appuyés sur des textes d'histoire comme l'Historia Gothorum d'Isidore de Séville afin de préciser ou de corriger des éléments de la liste qui ne répondaient pas à leur conception du passé. Le savoir se fonde ainsi sur des œuvres dont l'autorité est nettement supérieure à celle du genre mineur qu'est la liste. Celle-ci est refondue, réordonnée, adaptée à partir de ces autorités, de manière que surgisse un texte inédit.

La construction d'une liste repose sur un savoir-faire. L'auteur procède à un « recodage linguistique » pour réaliser ce nouveau support graphique $^{31}$. En outre, les qualités de la liste sont précieuses puisqu'elles permettent de condenser une pensée, ce qui permet d'isoler et d'identifier rapidement des informations utiles au lecteur. Dans le cas du manuscrit Paris, BnF, lat. 2718, l'auteur désirait combler ses lacunes en matière d'histoire mérovingienne. Il a fondé ses recherches sur des textes dont l'autorité ne faisait aucun doute, à savoir l'Histoire des Francs de Grégoire de Tours et la Chronique de Frédégaire, desquels il a extrait les informations qui satisfaisaient ses attentes. Une fois triés, classés, réduits et assemblés, ces éléments sont devenus un catalogue de noms de rois

\footnotetext{
${ }^{31}$ Jack Goody, La raison graphique..., op. cit., p. 193.
} 
inscrits sur le premier feuillet du manuscrit. Ce document était un aidemémoire concernant l'histoire mérovingienne. En somme, le moine de Saint-Martin a transformé des textes narratifs en un texte énumératif. Les deux exemples étudiés montrent que les listes de rois sont bien plus riches que ne le laisseraient penser leurs formes brèves et sèches. 